8 Kauppert C A, Dvorak I, Kollert F et al. Pulmonary Hypertension in obesity-hypoventilation syndrome. Resp Med 2013;107:2061-70.

9 Brown LK. Obesity hypoventilation syndrome. Curr Sleep Med Rep 2015;1:241-50.

10 Nolte JES, Koehler U, Sohrabi AK et al. Reversible severe pulmonary hypertension in obesity hypoventilation and Mohr Syndrome. Resp Med 2011;4:30-2.
Address for correspondence: Benoy N Shah, Cardiac Administration Offices, Level E North Wing, Southampton General Hospital, Tremona Road, Southampton S016 6YD, UK.

Email: benoy.shah@uhs.nhs.uk

\title{
Lesson of the month 2: Use of thrombolysis for ischaemic stroke in pregnancy - a case report and review of literature
}

\author{
Authors: Asim Khan, ${ }^{A}$ Paris Hosseini, ${ }^{B}$ Branimir Nevajda ${ }^{C}$ and Sami Khan ${ }^{D}$
}

A nine-week pregnant 33-year-old female presented with sudden-onset right-sided hemiparesis, hemisensory loss, dysarthria and homonymous hemianopia. She was known to have eleven previous miscarriages and used recreational drugs. A CT-head was unremarkable. The patient was subsequently diagnosed with an acute thromboembolic infarct and chose alteplase after counselling. During thrombolysis an ultrasound identified a foetus with no concerning features.

A post-thrombolysis $C T$ revealed a left-sided posterior cerebral infarct. CT-venography, carotid Doppler, 72-hour Holter monitor, thrombophilia and an autoimmune screen were all normal. A transthoracic echocardiogram demonstrated a mobile intra-atrial septum with a patent foramen ovale confirmed on bubble echocardiogram. Three days postthrombolysis the patient requested a termination of pregnancy. A subsequent transvaginal ultrasound demonstrated a missed miscarriage for which the patient underwent evacuation of retained products of conception. Multidisciplinary care ensured that her sensorimotor deficit resolved grossly with only mild dysarthria and right-hand fine motor incoordination.

\section{Case history}

A 33-year-old female in the ninth week of gestation presented with sudden-onset right-sided weakness, numbness and speech difficulty. Her previous medical history included eleven

Authors: ${ }^{\mathrm{A}}$ core medical trainee, Basildon University Hospital, Basildon and Thurrock NHS Foundation Trust; ${ }^{\mathrm{B}}$ medical student, University College London Medical School, University College London; ' neurology and stroke consultant, Basildon University Hospital, Basildon and Thurrock NHS Foundation Trust; ${ }^{D}$ radiology consultant, Basildon University Hospital, Basildon and Thurrock NHS Foundation Trust

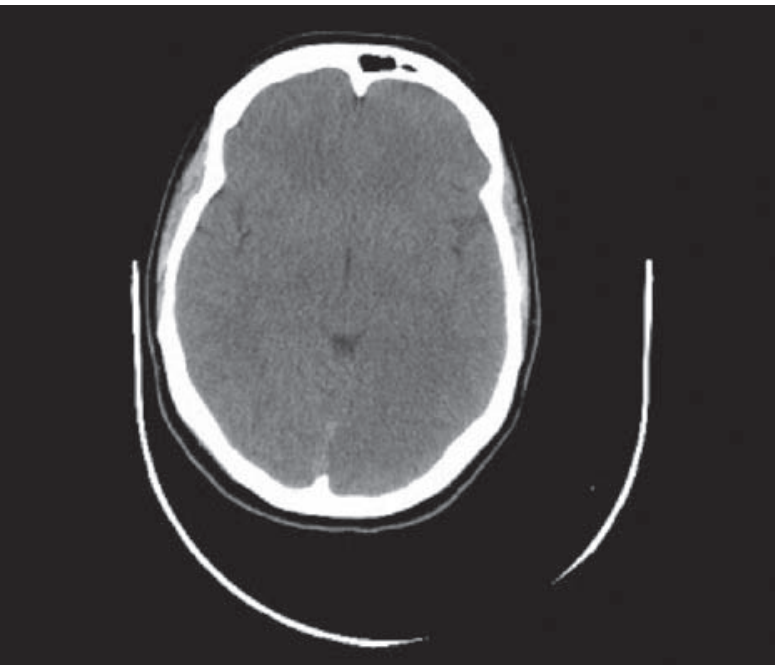

Fig 1. Initial CT-head pre-thrombolysis. Axial view. Upon retrospective analysis there is subtle evidence of the posterior cerebral infarct.

miscarriages of unknown cause. She had a ten-pack year smoking history and also used cocaine, cannabis, heroin and amphetamine recreationally.

Her vital observations and capillary blood glucose were normal. She was not confused. Speech assessment revealed dysarthria. Cranial nerve examination demonstrated a rightsided facial nerve palsy and homonymous hemianopia. Motor function in the limbs confirmed right-sided hemiparesis with a MRC grade of four and two in the upper and lower limbs respectively. Gait assessment was not possible. Tone was flaccid and reflexes were absent on the right-side with an equivocal right plantar response. There was a sensory deficit on the right-side. Cerebellar assessment demonstrated incoordination. 

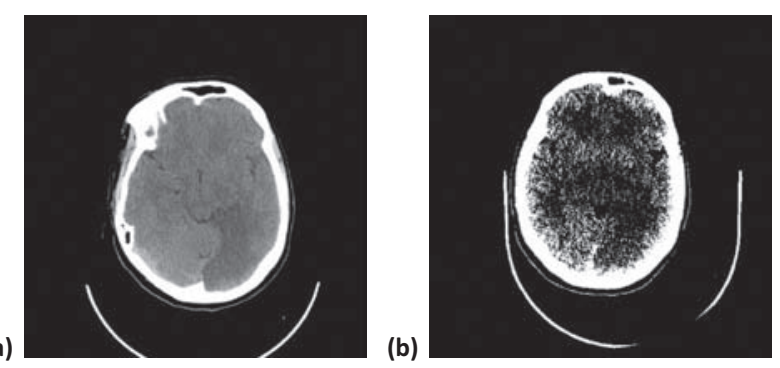

Fig 2. CT-head post-thrombolysis. (a) Axial view and (b) window view. Leftsided posterior cerebral infarct is demonstrated.

Neurological assessment of the left-side and examination of her other major organ systems was unremarkable.

The patient underwent an urgent non-contrast CT-brain which revealed no intracerebral haemorrhage or spaceoccupying lesion (Fig 1). The patient was subsequently diagnosed with an acute left-sided posterior cerebral thromboembolic infarct.

Given a lack of absolute contraindications, the patient and her family were counselled on the benefits and risks of thrombolysis compared to aspirin alone both to the patient and her foetus. Thrombolectomy was not considered because of the lack of availability within the local region. After deliberation the patient opted for thrombolysis which was supported by her family and the obstetric team. Alteplase was commenced two hours after initial symptom onset. During thrombolysis an abdominal ultrasound identified a foetal pole with no concerning features. Initial investigations including ECG, chest radiograph and blood tests (blood count differential, renal and liver function, electrolytes, C-reactive peptide, serum glucose, cholesterol and thyroid function) were entirely normal.

A repeat CT-brain post-thrombolysis demonstrated a wellestablished posterior cerebral infarct (Fig 2). A simultaneous CT-venogram excluded venous sinus thrombosis. Further investigations were performed to identify the underlying cause of the stroke, including a carotid Doppler, which was normal. A transthoracic echocardiogram revealed a mildly mobile inter-atrial septum with no obvious shunt. An autoimmune screen for connective tissue disease including anti-phospholipid syndrome, and a thrombophilia screen proved unremarkable.

At 24 hours post-thrombolysis the patient was commenced on aspirin. Statins were not initiated during the pregnancy because of risk of congenital malformations. The patient was given lifestyle advice including referral to a smoking cessation clinic and to the illicit drugs liaison team. At day three postthrombolysis the patient requested a termination of pregnancy. A transvaginal ultrasound revealed a nine-week-old foetus with no heartbeat consistent with a missed miscarriage, and a hypoechoic area adjacent to the gestation sac suggestive of haemorrhage. The patient subsequently underwent an evacuation of retained products of conception.

Multidisciplinary care ensured that by day six postthrombolysis the patient had improved neurologically. She was left with mild fine motor incoordination in the right hand, mild dysarthria and right homonymous hemianopia. She was however mobilising independently and self-caring. After two weeks post-thrombolysis aspirin was replaced with life-long clopidogrel. At a follow-up review three months

\begin{tabular}{l} 
Box 1. Risk factors for thromboembolic stroke during \\
pregnancy. \\
\hline Pregnancy-related \\
Caesarean delivery \\
Pregnancy-induced hypertension \\
Pre-eclampsia/eclampsia \\
Post-partum infection \\
Post-partum cerebral angiopathy \\
Gestational trophoblastic disease \\
Amniotic fluid embolism \\
Non-pregnancy-related \\
Hypertension \\
Smoking \\
Valvular heart disease \\
Dyslipidaemia \\
Thrombophilia \\
Sepsis \\
Migraine-with-aura \\
Paradoxical emboli \\
Illicit drug use \\
Sickle cell disease \\
Highlighted in bold are risk factors seen in the patient in this case. \\
\hline
\end{tabular}

later the patient's neurological findings had not changed. A repeat autoimmune and thrombophilia screen both proved unremarkable. A 72-hour Holter monitor also demonstrated no significant arrhythmias. A bubble-echocardiogram however identified a patent foramen ovale (PFO) for which she awaits closure.

\section{Discussion}

Stroke during pregnancy is rare, with an estimated incidence of 10-20 per 100,000 deliveries. The majority of cases occur during the peri-partum or post-partum period; the overall risk during this period is increased compared to non-pregnant patients of the same age. ${ }^{1}$ Risk factors for thromboembolic stroke can be broadly divided into pregnancy- and non-pregnancy-related factors (Box 1). ${ }^{2}$ Our patient's risk factors were smoking, illicit drug use and PFO. PFO-related strokes in pregnancy may be more likely to occur in the first or second trimesters. ${ }^{3}$

Radiation exposure to the foetus from CT-imaging is minimised because of collimation. The overall radiation dose to the foetus is less than 0.01 microGrays which does not increase the risk of miscarriage, foetal malformations, learning disabilities or childhood malignancy (Table 1). Nonetheless, patients can wear abdominal lead guards to protect the foetus from radiation. In our patient we did not perform a MRI postthrombolysis because of a theoretical risk of teratogenicity via thermal damage in the first trimester. However this is more likely to occur with MRI machines operating above a 1.5 Tesla magnetic field strength. ${ }^{5}$ 


\begin{tabular}{|c|c|c|}
\hline Image & $\begin{array}{l}\text { Radiation dose } \\
\text { to foetus (mGy) }\end{array}$ & $\begin{array}{l}\text { Risk of childhood cancer } \\
\text { per examination }\end{array}$ \\
\hline CXR & $0.001-0.01$ & $<1$ in $1,000,000$ \\
\hline CT Head & $0.001-0.01$ & $<1$ in $1,000,000$ \\
\hline AXR & $0.1-1.0$ & 1 in 100,000 to 1 in 10,000 \\
\hline CTPA & $0.01-0.1$ & $\begin{array}{l}1 \text { in } 1,000,000 \text { to } 1 \text { in } \\
100,000\end{array}$ \\
\hline V/Q Scan & $0.1-1.0$ & 1 in 100,000 to 1 in 10,000 \\
\hline CT Chest/Liver & $0.1-1.0$ & 1 in 100,000 to 1 in 10,000 \\
\hline CT Abdomen & $1.0-10$ & 1 in 10,000 to 1 in 1000 \\
\hline CT-CAP & $10-50$ & 1 in 1000 to 1 in 200 \\
\hline
\end{tabular}

Management of thromboembolic stroke during pregnancy is complex. Traditionally, thrombolysis was considered an absolute contraindication because of foetal haemorrhagic risks, especially during the peri-partum period. ${ }^{6}$ However thrombolytic drugs such as alteplase can be given safely in pregnancy. ${ }^{7-9}$ The drug is too large to cross the placenta so is not known to be teratogenic. ${ }^{9}$ The efficacy of alteplase in treating thromboembolic stroke during pregnancy is similar to that seen in non-pregnant women. However, the risk of haemorrhage including intracerebral and placental haemorrhage is increased, especially in the peri-partum period; the risk of placental haemorrhage can be reduced through catheter-driven thrombolysis. ${ }^{9,10}$ Overall, there is no increased risk of mortality. ${ }^{10}$ Thrombolectomy can also be considered especially for women in the peri-partum period.

Involvement of the obstetric team, particularly for women in their third trimester, is important as emergency delivery of the foetus may be required should complications ensue. ${ }^{9,10}$

Our patient was thrombolysed after counselling the patient on the advantages and disadvantages of each treatment. Despite the extent of her infarct the patient made a positive recovery and was left with minor impairment. She did however miscarry which could have been secondary to alteplase-induced placental haemorrhage. Nonetheless, her previous history of multiple spontaneous miscarriages and lifestyle choices were confounding factors.

\section{Conclusion}

Stroke can affect any subpopulation group including pregnant women and thrombolysis should be considered on a case-tocase basis. Involvement of the obstetric team is important, especially for women in the third trimester, where treatment complications may necessitate urgent foetal delivery. Finally, in any scenario the mother's health and safety should always take priority over that of the foetus.

\section{Acknowledgements}

Patient consent was obtained for publication of the article.

\section{References}

1 Kittner SJ, Stern BJ, Feeser BR et al. Pregnancy and the risk of stroke. N Engl J Med 1996;335:768-74.

2 Wabnitz A, Bushnell C. Migraine, cardiovascular disease, and stroke during pregnancy: systematic review of the literature. Cephalalgia 2015;35:132-9.

3 Chen L, Deng W, Palacios I, Inglessis-Azuaje I, McMullin D, Zhou D, et al. Patent foramen ovale (PFO), stroke and pregnancy. J Investig Med 2016; 64:992-1000.

4 Wall BF, Meara JR, Muirhead CR et al. Protection of pregnant patients during diagnostic medical exposures to ionising radiation. London: Royal College of Radiologists, 2009.

5 Medicines and Healthcare Products Regulatory Agency. Safety guidelines for magnetic resonance imaging equipment in clinical use. London: MHRA, 2015.

6 Murugappan A, Coplin WM, Al-Sadat AN et al. Thrombolytic therapy of acute ischemic stroke during pregnancy. Neurology 2006;66:768-70.

7 Tassi R, Acampa M, Marotta G et al. Systemic thrombolysis for stroke in pregnancy. Am J Emerg Med 2013;31:448.

8 Selim MH, Molina CA. The use of tissue plasminogen-activator in pregnancy. Stroke 2013;44:868-9.

9 Leonhardt G, Gaul C, Nietsch HH et al. Thrombolytic therapy in pregnancy. J Thromb Thrombolysis 2006;21:271-6.

10 Leffert LR, Clancy CR, Bateman BT et al. Treatment patterns and short-term outcomes in ischemic stroke in pregnancy or postpartum period. Am J Obstet Gynecol 2016;214:723.

Address for correspondence: Dr Asim Khan, Basildon University Hospital, Nethermayne, Basildon, Essex SS16 5NL Email: akhan2710@doctors.org.uk 